\title{
Wigner Function Description of the A.C.-Transport Through a Two-Dimensional Quantum Point Contact
}

\author{
Igor E. Aronov ${ }^{1,2}$, Gennady P. Berman ${ }^{1,3}$, David K. Campbell ${ }^{4}$, \\ and Sergey V. Dudiy ${ }^{2}$ \\ 1 Theoretical Division and the CNLS, Los Alamos National Laboratory, Los Alamos, New \\ Mexico, 87545, U.S.A., \\ 2 Institute for Radiophysics and Electronics, National Acadamy of Sciences of Ukraine, 12 Acad. \\ Proskura St., 310085, Kharkov, Ukraine, \\ ${ }^{3}$ Kirensky Institute of Physics, 660036, Krasnoyarsk, Russia, \\ ${ }^{4}$ Department of Physics, University of Illinois at Urbana-Champaign, 1110 West Green St., \\ Urbana, IL 61801-3080, U.S.A.
}

\begin{abstract}
We have calculated the admittance of a two-dimensional quantum point contact (QPC) using a novel variant of the Wigner distribution function (WDF) formalism. In the semiclassical approximation, a Boltzman-like equation is derived for the partial WDF describing both propagating and nonpropagating electron modes in an effective potential generated by the adiabatic QPC. We show that this quantum kinetic approach leads to the well-known stepwise behavior of the real part of the admittance (the conductance) [1], and of the imaginary part of the admittance (the emittance), in agreement with the latest results derived in [18, which is determined by the number of propagating electron modes.

It is shown, that the emittance is sensitive to the geometry of the QPC, and can be controlled by the gate voltage. We established that the emittance has contributions corresponding to both quantum inductance and quantum
\end{abstract}


capacitance. Stepwise oscillations in the quantum inductance are determined by the harmonic mean of the velocities for the propagating modes, whereas the quantum capacitance is a significant mesoscopic manifestation of the nonpropagating (reflecting) modes.

PACS numbers: 05.60; 72.10. Bgn; 72.30 


\section{INTRODUCTION}

Recent technological progress in manufacturing small-scale solid state structures has made possible the fabrication of devices involving two-dimensional electronic systems (2DES) in the quantum ballistic regime. One particular system that has attracted considerable attention is the quantum point contact (QPC) (see, e.g., [1 21]), which is fabricated by putting a split gate on the top of a $G a A s$ - AlGaAs heterostructure, thereby creating a narrow constriction in a two-dimensional electron gas (2DEG). Since in the ballistic regime the electrons do not experience any collisions, through the point contact is analogous to propagation of the electromagnetic wave through a waveguide. The width of the QPC which is controlled by the gate voltage can be of the same order of magnitude as the Fermi wavelength and governs the number of modes that can propagate through the constriction.

In QPC systems several experimental investigations [2 8] have demonstrated quantum coherent phenomena, including quantization of the d.c.-conductance versus the gate voltage (or the number of propagating modes through the QPC). The theory of this phenomenon [1.9 11] explains the d.c.-conductance quantization as a consequence of adiabatic transit of an electron wave through the QPC with smooth boundaries. In an adiabatic geometry (see Fig. 1), which is smooth on the scale of the Fermi wavelength, the longitudinal and transverse motion of electrons can be (approximately) separated in the Schrödinger equation [1, 10]. In this case the number of the transverse quantization modes is an adiabatic invariant, and the transverse energy plays the role of the potential energy for the one-dimensional longitudinal motion of each mode. Depending on whether the total energy of a given electron state is greater or less than the effective potential energy of a given mode, the the mode is propagating or non-propagating (see Fig. 2).

To date both experimental and theoretical studies of QPCs have been devoted mainly to investigations of the d.c.-conductance and the d.c.-transport. It is clear, however, that the investigation of the a.c.-transport can provide additional information, since a finite frequency introduces a new time-scale and may reveal qualitatively new effects, particularly if the new 
time-scale is of the order of other characteristic times of the system. The a.c.- conductance has been considered by M. Büttiker et al. [14 21], who established that the a.c.-transport is described by the a.c.-admittance $Y=1 / Z=G-i \omega \mathcal{E}$ at frequency $\omega$, where $Z$ is the impedance. (The authors of [22 25] considered the a.c. - kinetic response of the resonant tunnel junction.) The real part of the a.c.-admittance, $G$, is the conductance, and the imaginary part of $Y$, which is proportional to $\mathcal{E}$, was first introduced by M. Büttiker [17] as the emittance. In the important papers [14 21], the general expression for the electrochemical capacitance and for the displacement current were derived, and the steplike behavior of the QPC emittance in synchronism with the conductance steps, was established. Christen and Büttiker [19] also discussed the low-frequence QPC emittance of the quantized Hall conductors, and in [20] the authors used the scattering approach for the investigation of the nonlinear current-voltage characteristic of mesoscopic conductors. In papers [14 21], the emittance was expressed in terms of the geometric capacitance, transmission probability, and the densities of states of the "mesoscopic capacitor plates" [18].

In the present paper we extend the studies of the response of a QPC to an a.c. field by developing a simple method, based on the Wigner distribution function (WDF) formalism [26]227, for calculating the transport characteristics. Our approach allows us to represent the emittance in terms of the capacitance and the inductance, which are expressed in the explicit form through the microscopic characteristics. The effectiveness of the WDF approach to the modeling of small mesoscopic devices was demonstrated in Ref. [28,29]. In Section II, using the assumption of adiabaticity, we derive a Boltzmann-like quantum kinetic equation for a partial WDF describing transport in the quantum ballistic constriction. This equation allows us to treat the 2DES in a QPC in terms of classical trajectories for the effective 1D motion. The electron-electron Coulomb interaction is taken into account within the self-consistent field approximation. In Section III we demonstrate how the a.c.-admittance of the QPC can be calculates from the propagating and non-propagating (reflected) electron modes. Our approach recovers the quantized behavior as a function of gate voltage of the real part of the admittance (the conductance), consistent with previous calculations [1.10] using the 
Landauer formula [30]. Our approach also allows us to demonstrate that the emittance $\mathcal{E}$ has the negative part, which is a quantum inductance, to which all the propagating electron modes contribute and whose value is determined by the harmonic mean of the electron velocities in the quantized electron modes. The non-propagating electron modes determine the positive contribution to the emittance $\mathcal{E}$, which is a quantum capacitance, and which depends on a geometrical form of the QPC as controlled by the gate voltage. The conclusions are outlined in Section IV.

\section{KINETIC EQUATIONS IN A QUANTUM BALLISTIC CONSTRICTION}

To find the conductivity of a 2DES in a QBC form (see Fig. 1) taking into account both frequency dependence and spatial dispersion, we will apply the approach based on the Wigner distribution function (WDF) [26,27,

$$
f_{\vec{p}}^{W}(\vec{r})=\int d \vec{r}^{\prime} \operatorname{Tr}\left\{\hat{\rho} \exp \left[-\frac{i}{\hbar}\left(\vec{p}+\frac{e}{c} \vec{A}(\vec{r})\right) \vec{r}^{\prime}\right] \Psi^{+}\left(\vec{r}-\vec{r}^{\prime} / 2\right) \Psi\left(\vec{r}+\vec{r}^{\prime} / 2\right)\right\}
$$

Here $\hat{\rho}$ is the statistical operator of the system; $\Psi^{+}(\vec{r})$ and $\Psi(\vec{r})$ are, respectively, the

Fermi operators of creation and annihilation of particles at the point $\vec{r}$; and $\vec{A}$ is the vectorpotential of the electromagnetic field. When the characteristic scale of the spatial inhomogeneity exceeds both the radius of interaction among the particles and the electron's de Broglie wavelength, the kinetic equation for the WDF (1) assumes a form equivalent to the classical kinetic equation [27,

$$
\frac{\partial f_{\vec{p}}^{W}}{\partial t}+\vec{v} \frac{\partial f_{\vec{p}}^{W}}{\partial \vec{r}}+e\left\{\vec{E}+\frac{1}{c}[\vec{v}, \vec{B}]\right\} \frac{\partial f_{\vec{p}}^{W}}{\partial \vec{p}}=\hat{I}\left\{f_{\vec{p}}^{W}\right\}
$$

where as usual $\vec{E}$ and $\vec{B}$ are the electric and magnetic fields, and $e$ is the charge and $\vec{v}$ the velocity of conduction electrons. Eqn (2) is valid for the extended (in the $x-y$-plane) 2DES, when the typical scales of the inhomogeneity $\left(k^{-1}, d\right)$ are much smaller than a characteristic distance between the particles $n^{-1 / 2}: k, 1 / d \ll n^{-1 / 2}$, where $k^{-1}$ is the wavelength of the electromagnetic field, $d$ is a characteristic geometrical scale of the system, and $n$ is the 
density of the 2DEG. The characteristic distance between the particles is $\sim n^{-1 / 2}$ due to the weak screening in the 2DEG.

The collision integral, $\hat{I}\left\{f_{\vec{p}}^{W}\right\}$, in Eqn. (2), differs essentially from the classical collision integral, since the quantum transitions included in $\hat{I}\left\{f_{\vec{p}}^{W}\right\}$ reflect the character of the particle statistics and the distinction of the WDF from the classical one [27]. The equilibrium WDF sets the collision integral $\hat{I}\left\{f_{\vec{p}}^{W}\right\}$ to zero.

Using the definition of the WDF, we can express the charge density $\rho$ and the current density $\vec{j}$, respectively, as 26,27,

$$
\begin{aligned}
& \rho(t, \vec{r})=\frac{2 e}{(2 \pi \hbar)^{2}} \int d^{2} \vec{p} f_{\vec{p}}^{W}(\vec{r}), \\
& \vec{j}(t, \vec{r})=\frac{2 e}{(2 \pi \hbar)^{2}} \int d^{2} \vec{p} \vec{v} f_{\vec{p}}^{W}(\vec{r}) .
\end{aligned}
$$

Despite the evident analogy with the classical distribution function, it is well known that the WDF does not have an interpretation as the probability density, since it can take both positive and negative values, but the integrated values shown in Eqns. (3) and (4) have the usual physical meanings.

For a finite system, when a 2DEG is located in a bounded region (see Fig. 1) characterized by distances $d$ of the order of the Fermi wavelength, the left-hand side of the kinetic equation (2) changes its form. Using a standard procedure [26,27], one can obtain the kinetic equation for the WDF in the 2DES within the strip-like restricted region $|y|<d(x), d(x)=$ const, which can be written in the form,

$$
\begin{aligned}
& \frac{\partial f_{\vec{p}}^{W}}{\partial t}+\vec{v} \frac{\partial f_{\vec{p}}^{W}}{\partial \vec{r}}+e\left\{\vec{E}+\frac{1}{c}[\vec{v}, \vec{B}]\right\} \frac{\partial f_{\vec{p}}^{W}}{\partial \vec{p}}+ \\
& +\frac{4 \operatorname{sgn}(y)}{m \pi \hbar} \int_{-\infty}^{\infty} d p_{y}^{\prime} p_{y}^{\prime} \cos \left[\frac{2\left(p_{y}-p_{y}^{\prime}\right)}{\hbar}(d-|y|)\right] f_{p_{x}, p_{y}^{\prime}}^{W}=\hat{I}\left\{f_{\vec{p}}^{W}\right\},
\end{aligned}
$$

where $\operatorname{sgn}(y)$ is the sign function. The integral term in the left-hand side of Eqn. (5) arises from the transverse quantization. The presence of this term precludes the naive application 
of the classical treatment, based on the trajectories, for the solution of the kinetic equation (5) for the WDF.

If $d(x) \neq$ const, the kinetic equation for the WDF assumes an even more complicated form. To overcome these difficulties, we will invoke the adiabatical assumption [四] for the structure of the QPC shown in Fig. 1. Explicitly, we shall assume that the constriction is sufficiently long and smooth, the criterion

$$
d^{\prime}(x) \simeq d(x) / \tilde{L} \ll 1
$$

is met (where $2 \tilde{L}$ is the length of the constriction), that the transport is adiabatic. With this assumption, which has been discussed and analyzed in [1, 10], the variables in the Schrödinger equation can be separated, and the eigen-wave function can be written in the form,

$$
\psi_{n}(x, y)=\psi_{n}(x) \Phi_{n}[y, d(x)]
$$

where the transverse wave function

$$
\Phi_{n}(y)=\frac{1}{\sqrt{d(x)}} \sin \left\{\frac{\pi n[y+d(x)]}{2 d(x)}\right\} \theta\left[d^{2}(x)-y^{2}\right],
$$

should satisfy the boundary conditions:

$$
\left.\Phi_{n}(y)\right|_{y= \pm d(x)}=0
$$

and $\theta(x)$ is the Heavisaid single-step function.

One can then derive an effective Hamiltonian for the longitudinal wave function $\psi_{n}(x)$ as

$$
\hat{H}=-\frac{\hbar^{2}}{2 m} \partial_{x x}^{2}+\varepsilon_{n}(x)+e \phi(x) .
$$

In $(9) \phi(x, y)$ is an electric potential, and $\phi(x)$ is the averaged electric potential with respect to the transverse coordinate $y$,

$$
\phi(x)=\frac{1}{2 d(x)} \int_{-d}^{d} d y \phi(x, y)
$$


The electric potential $\phi(x, y)$ is assumed to by a smoothly varying function of the transverse coordinate $y$ within the constriction region $|y|<d(x)$. Due to the transverse quantization, the energy of the transverse motion $\varepsilon_{n}(x)$ in the Hamiltonian (9) has the form,

$$
\varepsilon_{n}(x)=\frac{\pi^{2} n^{2} \hbar^{2}}{8 m d^{2}(x)}
$$

With our assumptions, the transverse quantum number $n$ is an adiabatic integral of motion. Hence we can consider the motion of electrons in the QPC as for a set of effective one-dimensional electron systems enumerated by $n$. Each effective electron system is located in both the potential $\varepsilon_{n}(x)$ and the self-consistent electrical potential $\phi(x)$. We can introduce the partial WDF (PWDF) as

$$
f_{n}^{W}\left(x, p_{x}\right)=\int d x^{\prime} \exp \left(-\frac{i p_{x} x^{\prime}}{\hbar}\right) \operatorname{Tr} \hat{\rho} \Psi_{n}^{+}\left(x-x^{\prime} / 2\right) \Psi_{n}\left(x+x^{\prime} / 2\right) .
$$

Using (11), we can represent the WDF in the form,

$$
f_{\vec{p}}^{W}(\vec{r})=\sum_{n=1}^{\infty} f_{n}^{W}\left(x, p_{x}\right) \int_{-\infty}^{\infty} d y^{\prime} \exp \left(-\frac{i p_{y} y^{\prime}}{\hbar}\right) \Phi_{n, x}\left(y-y^{\prime} / 2\right) \Phi_{n, x}\left(y+y^{\prime} / 2\right) .
$$

We can derive the equation for the PWDF (11) with the use of the Wigner transformation 26,27 :

$$
\frac{\partial f_{n}^{W}}{\partial t}+v_{x} \frac{\partial f_{n}^{W}}{\partial x}+\left[-\frac{\partial \varepsilon_{n}(x)}{\partial x}+e E(x)\right] \frac{\partial f_{n}^{W}}{\partial p}(x, p)=\hat{I}\left\{f_{\vec{p}}^{W}\right\}
$$

where $p \equiv p_{x}$ and

$$
E(x)=-\frac{\partial \phi(x)}{\partial x}
$$

In terms of the PWDF the nonequilibrium charge density and current density can be defined as:

$$
\begin{aligned}
& \rho(x, y)=\sum_{n=1}^{\infty} \rho_{n}(x) \Phi_{n}^{2}(y), \\
& j(x, y)=\sum_{n=1}^{\infty} j_{n}(x) \Phi_{n}^{2}(y),
\end{aligned}
$$

where $\rho_{n}(x)$ and $j_{n}(x)$ are the partial charge and current densities: 


$$
\begin{gathered}
\rho_{n}(x)=\frac{e}{\pi \hbar} \int_{-\infty}^{\infty} d p\left[f_{n}^{W}(x, p)-f_{n}^{W(0)}(x, p)\right], \\
j_{n}(x)=\frac{e}{\pi \hbar m} \int_{-\infty}^{\infty} d p p f_{n}^{W}(x, p) .
\end{gathered}
$$

In $(16), f_{n}^{W(0)}(x, p)$ is the equilibrium PWDF.

The motivation for introducing the PWDF is now clear. In contrast to Eqn. (6), the kinetic equation (13) describing for the PWDF does have the form of a classical kinetic equation in the presence of an effective potential $\varepsilon_{n}(x)$. Hence the solution of this equation can be described by the characteristics, i.e., by the classical trajectories.

The formalism used in this paper is based on the assumption that the kinetic equation for the WDF can also involve the collision integral. It is well-known (see, e.g., Ref. [27]) that this can be realized at crystal periodicity violation, which is a source of electron scattering not distorting (or distorting weakly) the electron spectrum of the ideal crystal. In this way a weak disorder can be taken into account within the WDF formalism. Certainly, the impurity scattering in a form of the collision integral for the WDF must be treated self-consistently using, e.g., the self-consistent Born approximation, which is the simplest method that is free from divergences. In other words, the collision integral can be described in terms of the relaxation frequency depending on the electron energy. It is clear, that in the case when the current carriers have a high mobility, and if the frequencies of the electromagnetic field are sufficiently high, the approximation for the collision integral is justified. The forms of the electron-phonon and electron-impurity collision integrals are too complicated [27. However, we shall consider here the effects associated with the linear response to the electric field. In this case, the WDF can be found in a linear approximation with respect to the external electric field $\vec{E}$. It is well-known [27] that for describing the high-frequency effects $(\omega \gg \nu)$ in a sample with a high electron mobility, the collision integral can be treated in terms of the momentum relaxation frequency $\nu$, while the mean free path time is $1 / \nu$.

In other words, the collision integral in (13) includes quantum transitions [27 and intermixing of the different electron modes (the different PWDF). Below, we assume a quasi- 
ballistic regime of transport through the QPC, and will approximate the collision integral by a single momentum relaxation frequency,

$$
\hat{I}_{n}\left\{f_{\vec{p}}^{W}\right\}=-\nu\left[f_{n}^{W}(x, p)-f_{n}^{W(0)}\right]
$$

where $f_{n}^{W(0)}$ is the equilibrium PWDF. The equilibrium distribution function $f_{n}^{W(0)}$ within the adiabatic approximation is given by,

$$
\begin{gathered}
f_{n}^{W(0)}(x, p)=n_{F}\left\{\frac{p^{2} / 2 m+\varepsilon_{n}(x)-\mu}{T}\right\}, \\
n_{F}(x)=\left(1+e^{x}\right)^{-1} .
\end{gathered}
$$

The function $n_{F}(x)$ is the Fermi function with the effective chemical potential $\mu-\varepsilon_{n}(x)$, where $\mu$ is the equilibrium chemical potential of the 2DEG. The effective chemical potential varies smoothly as a function of the longitudinal coordinate $x$. In this paper we are interested in the linear response, so we expand the PWDF about its equilibrium form

$$
f_{n}^{W}(x, p)=f_{n}^{W(0)}(x, p)+f_{n}(x, p)
$$

The kinetic equation linearized in the electric field $E(x, t)=E(x) \exp (-i \omega t)$, becomes

$$
\frac{p}{m} \frac{\partial f_{n}}{\partial x}-\frac{\partial \varepsilon_{n}(x)}{\partial x} \frac{\partial f_{n}}{\partial p}+(\nu-i \omega) f_{n}=-e E \frac{\partial f_{n}^{W(0)}}{\partial p}
$$

The natural method for solving the kinetic equation (21) is the method of characteristics. The characteristics of this equation are the phase trajectories of a one-dimensional motion in the potential $\varepsilon_{n}(x)$, which is determined from the integral of motion, viz. the total energy $\varepsilon$ :

$$
\varepsilon=\frac{p^{2}}{2 m}+\varepsilon_{n}(x)=\text { const }
$$

We will consider a reflection symmetric QPC, i.e., $d(x)=d(-x)$. For this case the phase portrait is shown in Fig. 2. The heavy lines in Fig. 2 denote the separatrix, which passes 
through the hyperbolic point $p=0, x=0$ and separate the phase space into four regions, within which four sets of phase trajectories exist.

The regions of propagating trajectories $\left(\varepsilon>\varepsilon_{n}(0)\right)$ occupy the regions (see Fig. 2):

$$
\text { 1) } \varepsilon>\varepsilon_{n}(0), p>0 ; \text { and 2) } \varepsilon>\varepsilon_{n}(0), p<0 \text {. }
$$

The regions of non-propagating (reflecting) trajectories $\left(\varepsilon<\varepsilon_{n}(0)\right)$ :

$$
\text { 3) } \varepsilon<\varepsilon_{n}(0), x>0 ; \text { and 4) } \varepsilon<\varepsilon_{n}(0), x<0 \text {. }
$$

Within each region, one can find the solution of the kinetic equation for the PWDF and derive the general formula for the partial charge $\rho_{n}$ and the current densities $j_{n}$. Here we consider the most interesting case, when the temperature is very low $(T \rightarrow 0, T \ll \mu)$, so that we have a clear separation between propagating $\left(\varepsilon_{n}(0)<\mu\right)$ and reflecting $\left(\varepsilon_{n}(0)>\mu\right)$ channels.

For the "open" (i.e., propagating) channels:

$$
\begin{gathered}
\rho_{n}(x)=\frac{2 e^{2}}{h} \frac{1}{v_{n}(x)} \int_{-L}^{L} d x^{\prime} E\left(x^{\prime}\right) \operatorname{sgn}\left(x-x^{\prime}\right) \exp \left[i \omega^{*} \tau_{n}\left(x, x^{\prime}\right) \operatorname{sgn}\left(x-x^{\prime}\right)\right], \\
j_{n}(x)=\frac{2 e^{2}}{h} \int_{-L}^{L} d x^{\prime} E\left(x^{\prime}\right) \exp \left[i \omega^{*} \tau_{n}\left(x, x^{\prime}\right) \operatorname{sgn}\left(x-x^{\prime}\right)\right]
\end{gathered}
$$

where $\omega^{*}=\omega+i \nu, v_{n}(x)=\sqrt{(2 / m)\left[\mu-\varepsilon_{n}(x)\right]}$, and

$$
\tau_{n}\left(x, x^{\prime}\right)=\int_{x^{\prime}}^{x} \frac{d x^{\prime \prime}}{v_{n}\left(x^{\prime \prime}\right)} .
$$

For the closed channels (reflecting modes)

$$
\begin{gathered}
\rho_{n}(x)=\frac{2 e^{2}}{h} \frac{\operatorname{sgn}(x)}{v_{n}(x)} \int_{x_{n}}^{L} d x^{\prime} E\left(x^{\prime} \operatorname{sgn}(x)\right) \times \\
\left\{\operatorname{sgn}\left(|x|-x^{\prime}\right) \exp \left[i \omega^{*} \tau_{n}\left(|x|, x^{\prime}\right) \operatorname{sgn}\left(|x|-x^{\prime}\right)\right]-\exp \left[i \omega^{*}\left(\tau_{n}\left(|x|, x_{n}\right)+\tau_{n}\left(x^{\prime}, x_{n}\right)\right)\right]\right\} \theta\left(|x|-x_{n}\right) \\
j_{n}(x)=\frac{2 e^{2}}{h} \int_{x_{n}}^{L} d x^{\prime} E\left(x^{\prime} \operatorname{sgn}(x)\right) \times
\end{gathered}
$$




$$
\left\{\exp \left[i \omega^{*} \tau_{n}\left(|x|, x^{\prime}\right) \operatorname{sgn}\left(|x|-x^{\prime}\right)\right]-\exp \left[i \omega^{*}\left(\tau_{n}\left(|x|, x_{n}\right)+\tau_{n}\left(x^{\prime}, x_{n}\right)\right)\right]\right\} \theta\left(|x|-x_{n}\right) .
$$

Here $x_{n}$ is the absolute value of the critical (turning) point, which is determined by the condition

$$
\varepsilon_{n}\left(x_{n}\right)=\mu \text {. }
$$

From Eqns. (23), (24) and (26), (27), it is apparent that the transport through a QPC is described by highly nonlocal (integral) operators. This suggests that the charge and current densities at a given point $x$ are influenced by the electrical field within the whole conductor. Thus, the PWDF formalism allowed us to derive the charge and the current densities as nonlocal operators with respect to the electric field.

\section{THE ADMITTANCE OF THE QPC}

Our formulation of the kinetic equation for the PWDF allows us to describe the adiabatic transport through a QPC. Using Eqns. (23), (24) and (26), (27), we can calculate the charge and current densities in the QPC, once the field distribution within the QPC is given. Of particular experimental interest is the calculation of the frequency-dependent the admittance of the QPC, the behavior of which reveals more detailed information than any static characteristics.

It is well-known that the static conductance is fully specified by the potential difference (bias voltage) between the right and left reservoirs and that the while detailed electrical potential profile does not influence it significantly [四]. This result was derived using the Landauer formalism [10,30] when the conductance was defined by the matrix of the transmission coefficients of the electrons corresponding to the different propagating chennals. We can readily show that this result also follows immediately from our PWDF approach. In the ballistic regime, when

$$
L \ll l,
$$


( $2 L$ is the distance between the reservoirs, $l$ is the mean free path), for $\omega, \nu \rightarrow 0$, we find for the propagating modes (open channels):

$$
j_{n}=\frac{2 e^{2}}{h} V, \quad V=\int_{-L}^{L} d x E(x),
$$

and for the non-propagating modes (closed channels):

$$
j_{n}=0
$$

Using Eqn. (15), we obtain the for the total current flowing through the QBC the result

$$
I=\int_{-\infty}^{\infty} d y j(y)
$$

Hence the static conductance assumes the familiar form [1]:

$$
G=\frac{I}{V}=\frac{2 e^{2}}{h} \mathcal{N}
$$

where $\mathcal{N}$ is the number of the open channels:

$$
\mathcal{N}=\left[\frac{2 k_{F} d(0)}{\pi}\right] ; \quad \hbar k_{F}=\sqrt{2 m \mu} .
$$

Here the brackets [...] stand for the integral part of the enclosed expression. From these equations it is clear that the static conductance does not depend on the details of the smooth function, $d(x)$.

More generally, we can use the formalism of the PWDF to calculate the admittance at the frequency $\omega$. From formulas (24) - (28) one can see that the partial current $j_{n}$ is a function of the longitudinal coordinate $x$ at $\omega \neq 0$. The continuity equation

$$
\operatorname{div} \vec{j}+\frac{\partial \rho}{\partial t}=0
$$

in the QPC at $\omega \neq 0$ takes a form:

$$
\sum_{n=1}^{\infty} \frac{\partial}{\partial x}\left\{j_{n}-i \omega \int_{-L}^{x} d x^{\prime} \rho_{n}\left(x^{\prime}\right)\right\}=\frac{\partial}{\partial x}\left\{I_{t o t}\right\}=0
$$

where 


$$
I_{\text {tot }}=\sum_{n=1}^{\infty}\left\{j_{n}-i \omega \int_{-L}^{x} d x^{\prime} \rho_{n}\left(x^{\prime}\right)\right\} .
$$

Note that the total current $I_{t o t}$, which includes the current density $\sum_{n=1}^{\infty} j_{n}(x)$ and displacement current $-i \omega \sum_{n=1}^{\infty} \int_{-L}^{x} d x^{\prime} \rho_{n}\left(x^{\prime}\right)$ is independent of the longitudinal coordinate $x$. From the form of Eqn. (36) it is easy to see that the displacement current vanishes within the left reservoir, so the total current is

$$
I_{t o t}=\sum_{n=1}^{\infty} j_{n}(-L)
$$

and the admittance can be determined as

$$
Y=\frac{I_{t o t}}{V}=\frac{1}{V} \sum_{n=1}^{\infty} j_{n}(-L)
$$

In the general case, we should determine the field $E(x)$ within the QBC from the Maxwell equations and afterwards calculate the admittance. Here we consider the long-wavelength approximation, in which

$$
v_{n}^{*} \gg \omega L_{n}
$$

Here $v_{n}^{*}$ is the typical velocity for the electrons of the $n$-th channel, $L_{n}$ characterizes the length of a region for each channel. For the open (propagating) modes $L_{n}$ is the distance between the reservoirs $\left(L_{n} \sim 2 L\right)$ and

$$
v_{n}^{*}=v_{n}(0)
$$

For non-propagating modes (closed channels) modes, $L_{n}$ is twice the distance between the turning point (28) and the nearest reservoir $\left(L_{n} \sim 2\left(L-x_{n}\right)\right)$. The typical velocity in this case is

$$
v_{n}^{*}=\frac{2 v_{F}}{\tilde{L}} \sqrt{x_{n}\left(L-x_{n}\right)}, \quad v_{F}=\sqrt{\frac{2 \mu}{m}},
$$

where $2 \tilde{L}$ is the length of the constriction. 
The condition (39) means that the field changes only slightly during the time that it takes an electron to transit through the QPC. Hence Eqn. (39) is the condition for weak frequency dispersion of the conductivity. To calculate the current of the propagating modes (open channels), we can approximate the velocity $v_{n}$ as

$$
v_{n}(x) \simeq v_{n}(0)=v_{n}^{*},
$$

and for the reflecting modes:

$$
v_{n}(x) \simeq v_{n}^{*} \sqrt{\frac{|x|-x_{n}}{L-x_{n}}} .
$$

We approximate the form of the QBC (as in [12]):

$$
d(x)=d_{0} \exp \left[(x / \tilde{L})^{2}\right]
$$

Using this approximation, we get for the open channels:

$$
j_{n}(-L)=\frac{2 e^{2}}{h}\left(1+i \frac{\omega L}{v_{n}^{*}}\right) V
$$

and for the closed channels:

$$
j_{n}(-L)=-i \omega \frac{8 e^{2}}{h} \frac{\left(L-x_{n}\right)}{v_{n}^{*}} \int_{x_{n}}^{L} d x^{\prime} E\left(x^{\prime}\right) \sqrt{\frac{x^{\prime}-x_{n}}{L-x_{n}}} .
$$

Consistent with our choice of a reflection symmetric $d(x)$, let us assume that the electric field inside the QPC is reflection symmetric, $E(x)=E(-x)$. In this case, the contribution of the open channels is determined by the total voltage $V$ and is independent of the detailed profile of the electrical potential inside the QPC. Thus, we can write the admittance in the form:

$$
Y=G-i \omega \mathcal{E}
$$

where $G=\left(2 e^{2} / h\right) \mathcal{N}$ is the static conductance. The emittance $\mathcal{E}$ of the QPC is given by the expression

$$
\mathcal{E}=-G \frac{L}{\bar{v}^{(o)}}+\frac{16}{3} \frac{e^{2}}{h} \sum_{n=\mathcal{N}+1}^{\mathcal{N}+\tilde{\mathcal{N}}} \frac{\xi_{n}}{v_{n}^{*}}\left(L-x_{n}\right) .
$$


Here $\bar{v}^{(o)}$ is the harmonic mean of the velocities $v_{n}^{*}$ in the open channels (42):

$$
\frac{1}{\bar{v}^{(o)}}=\frac{1}{\mathcal{N}} \sum_{n=1}^{\mathcal{N}} \frac{1}{v_{n}^{*}} \text {. }
$$

The integer $\tilde{\mathcal{N}}$ determines the number of the closed channels:

$$
\tilde{\mathcal{N}}=\left[\frac{2 k_{F} d_{0}}{\pi} \exp \left[(L / \tilde{L})^{2}\right]\right]-\mathcal{N}
$$

with $2 L$ being the distance between the reservoirs. The discrete value $\xi_{n}$ characterizes the relative bias of voltage in the region $\left(x_{n}, L\right)$ filled with the electrons of the $n^{\text {th }}$ reflecting channel:

$$
\xi_{n}=\frac{3}{2} \int_{x_{n}}^{L} d x^{\prime} \frac{E(x \prime)}{V} \sqrt{\frac{x^{\prime}-x_{n}}{L-x_{n}}} .
$$

¿From Eqn. (48) it follows immediately that the contribution of the reflecting modes to the emittance $\mathcal{E}$ is positive, whereas the contribution of the propagating modes is negative. This observation allows us to express our results concisely in terms of the equivalent circuit shown in Fig. 3. The admittance of the circuit is

$$
Y=G-i \omega\left(C-\Lambda G^{2} / c^{2}\right)
$$

with

$$
\omega C \ll G, \quad \omega \Lambda \ll c^{2} G^{-1} .
$$

The effective inductance in Eqn. (52) is

$$
\Lambda=\frac{c^{2} L}{G \bar{v}^{(o)}}
$$

and the effective capacitance is

$$
C=\frac{16}{3} \frac{e^{2}}{h} \sum_{n=\mathcal{N}+1}^{\mathcal{N}+\tilde{\mathcal{N}}} \frac{\xi_{n}}{v_{n}^{*}}\left(L-x_{n}\right) .
$$

Note, that Eq. (52) coincides with the general expression for the emittance derived in [18 (see Eq. (7) in [18]), where the emittance was expressed in terms of the geometric capacitance, transmission probability, and the densities of states of the "mesoscopic capacitor 
plates" . Our description allowed us to represent the emittance in terms of the inductance (53), and the capacitance (54), which are expressed in the explicit form through the microscopic characteristics such as the harmonic mean of the velocities of the open channels (inductance), and the relative bias of voltage of the QPC $\xi_{n}(51)$, velocities $v_{n}^{*}$, and the values of the turning points $x_{n}$ (see (54)). It is easy to see, that the capacitance (54) and the inductance (53) demonstrate the stepwise behavior as the functions of the gate voltage. This stepwise behavior of the emittance, as it was pointed out in 18, is in a synchronism with the conductance steps, and is determined by the number of open (or closed) channels in the QPC.

We can readily show what the emittance is a stepwise function of the gate voltage. When the gate voltage approaches a point for which $2 k_{F} d / \pi$ is integer, and one more mode opens (or closes), the inductance and the capacitance in the expressions (53) and (54) increase to infinity.

In this case, the condition (39) is violated, and the contribution of these points to the admittance must be calculated separately. Let us analyze the asymptotic behavior of the emittance in this case. The approximation (42),(43) is justified only if for all modes the parameter

$$
\gamma_{n}=\left(\mu-\varepsilon_{n}(0)\right) / \varepsilon_{n}(0)
$$

is not too small. The situation when $\gamma_{n}$ becomes small for the $n_{0}^{\text {th }}$ mode $\left(n_{0}=\mathcal{N}, \mathcal{N}+1\right)$ means that the corresponding mode is near to the point where it transforms from propagating to non-propagating, or vice versa. When $\left|\gamma_{\mathcal{N}}\right| \ll 1$ (for an open channel), we find that in an inequality (39) and in Eq. (49) the typical velocity for $n=\mathcal{N}$ is

$$
v_{\mathcal{N}}^{*} \simeq v_{F} \frac{L}{\tilde{L}} \frac{\sqrt{2}}{\ln \left(4 L^{2} / \tilde{L}^{2}\left|\gamma_{\mathcal{N}}\right|\right)} .
$$

If $\left|\gamma_{\mathcal{N}+1}\right| \ll 1$ (for a closed channel) then in an inequality (39) and in Eqs (48), (53):

$$
v_{\mathcal{N}+1}^{*} \simeq \frac{16}{3} v_{F} \frac{L}{\tilde{L}} \frac{\sqrt{2}}{\ln \left(4 L^{2} / \tilde{L}^{2}\left|\gamma_{\mathcal{N}+1}\right|\right)} .
$$

Hence the contribution of the $\mathcal{N}^{\text {th }}$ mode to the inductance is 


$$
\Lambda_{\mathcal{N}} \simeq \frac{c^{2}}{G^{2}} \frac{2 e^{2}}{h} \frac{\tilde{L}}{v_{F} \sqrt{2}} \ln \left(\frac{4 L^{2}}{\left|\gamma_{\mathcal{N}}\right| \tilde{L}^{2}}\right)
$$

And the contribution of the $(\mathcal{N}+1)^{s t}$ mode to the capacitance is

$$
C_{\mathcal{N}+1} \simeq \frac{e^{2}}{h} \frac{\tilde{L}}{v_{F} \sqrt{2}} \ln \left(\frac{4 L^{2}}{\left|\gamma_{\mathcal{N}+1}\right| \tilde{L}^{2}}\right)
$$

If a channel opens (closes), and $\gamma_{n} \rightarrow 0$, there can be a case of strong frequency and spatial dispersion. Because of this, at these points the system can not be treated in terms of effective inductance and capacitance. (Note, that in Eqs. (55)-58), when $\gamma_{n} \rightarrow 0$, the

modules $\left|\gamma_{\mathcal{N}}\right|$ and $\left|\gamma_{\mathcal{N}+1}\right|$ should be substituted by $\sqrt{\gamma_{n}^{2}+(L / l)^{2}}$, where $l$ is the electron's mean free path in the ballistic quantum constriction, $L / l \ll 1$.)

The emittance is described by the parameters of different nature. The inductance $\Lambda$ is determined by the velocities $v_{n}$ for the open channels, and the capacitance $C$ is mainly determined by the distribution of the electrical field as well as by the location of the turning points (28). The mesoscopic emmitance can be controlled by the gate voltage.

\section{CONCLUSIONS}

We have developed a new approach, based on a partial Wigner distribution function, to analyze electron a.c electron transport properties of a quantum point contact. Treating the quantum ballistic constriction in the adiabatic approximation, we derived a Boltzman-like equation for the partial Wigner distribution function in an effective potential brought about by the quantized transverse modes. We analyzed this equation in terms of propagating and reflecting trajectories in the quasiclassical approximation.

Our results establish that the a.c electron transport depends directly on the the number of propagating and reflecting modes and that certain features are sensitive to the form of the distribution of the electric field in the QPC. In particular, the real part of the admittance (the conductance) is determined by the number of propagating electron modes, and does not depend on the spatial distribution of the electric field inside the QPC [1]. The imaginary part of the admittance (the emittance) exhibits stepwise oscillations as a function of the 
gate voltage and consists of two parts: the quantum inductance and the quantum capacitance. The quantum inductance is determined by the harmonic mean of the velocities for the propagating electron modes. The quantum mesoscopic capacitance is specified by the reflecting modes that are very sensitive to the geometry of the QPC. The emmitance can be controlled by the gate voltage. Therefore, the measurements of the admittance can be more informative than the measurements of the static conductance.

It is important to stress that the effective quantum inductance and capacitance, and the equivalent circuit, are concepts valid within our linear response, low-frequency approximation. For the high-frequency case, and when new propagating and non-prpagating modes can appear or disappear, the frequency dispersion of the admittance is more complicated than the linear one given by the equivalent circuit of Eq. (52). This case must be considered using the self-consistent Maxwell equations for the electric field in the QPC. We are presently investigating this problem.

\section{ACKNOWLEDGMENTS}

We are grateful to L.I. Glazman, D.K. Ferry, R. Akis and G.D. Doolen for fruitful discussions. We thank the Theoretical Division and the Center for Nonlinear Studies of the Los Alamos National Laboratory for hospitality during the completion of this work. This research was supported in part by the Linkage Grant 93-1602 from the NATO Special Programme Panel on Nanotechnology, by the Grant 94-02-04410 of the Russian Fund for Basic Research, by the INTAS Grant No. 94-3862, and by the Ukrainian Committee for Science and Technology (project No. 2.3/19 "Metal"). 


\section{REFERENCES}

[1] L. I. Glazman, G. B. Lesovik, D. E. Khmelnitskii, and R. Shekhter, JETP Lett., 48, 238 (1988).

[2] C. W. J. Beenakker and H. van Hasten, In: Solid State Physics, Eds: H. Ehrenreich and D. Turnbull (Academic, San Diego, 1991), Vol. 44, p. 1.

[3] B. J. van Wees, H. van Houten, C. W. J. Beenakker, J. G. Williamson, L. P. Kouwenhoven, D. van der Mare and C. T. Foxon, Phys. Rev. Lett., 60, 848 (1988).

[4] D. A. Wharam, T. J. Thornton, R. Newbury, M. Pepper, H. Ahmed, J. E. F. Frost, G. G. Hasko, D. C. Peacock, D. A. Ritchie, and G. A. C. Jones, J. Phys. C, 21, L209 (1988).

[5] B. J. van Wees, L. P. Kouwenhoven, H. van Houten, C. W. J. Beenakker, J. E. Moori, C. T. Foxon, and J. J. Harris, Phys. Rev. B, 38, 3625 (1988).

[6] N. K. Patel, J. T. Nicholls, L. Martin - Moreno, M. Pepper, J. E. F. Frost, D. A.Ritchie, and G. A. C. Jones, Phys. Rev. B, 44, 13549 (1991).

[7] R. Taboryski, A. K. Geim, and P. E. Lindelof, Superlatt. Microstruc., 12, 137 (1992);

R. Taboryski, A. K. Geim, M. Persson, and P. E. Lindelof, Phys. Rev. B, 49, 7813 (1994).

[8] T. M. Eiles, J. A. Simmons, M. E. Sherwin, and J. F. Klem, Phys. Rev. B, 52, 10756 (1995).

[9] L. I. Glazman and M. Jonson, Phys. Rev. B, 41, 10686 (1989).

[10] A. Yacoby and Y. Imry, Europhys. Lett., 11, 663 (1990).

[11] I. E. Aronov, M. Jonson, and A. M. Zagoskin, Phys. Rev. B, 50, 4590 (1994).

[12] A. Grincwaig, M. Jonson, and R. I. Shekhter, Phys. Rev. B, 49, 7557 (1994);

L. Gorelik, A. Grincwaig, V. Kleiner, R.Shekhter, and M. Jonson, Phys. Rev. Lett., 73, 
2260 (1994); A. Grincwaig, L. Gorelik, V. Kleiner, and R. I. Shekhter, Phys. Rev. B, 52, $12168(1995)$.

[13] F. Hekking and Yu. V. Nazarov, Phys. Rev. B, 44, 11506 (1991); ibid 44, 9110 (1991).

[14] M. Büttiker, H. Thomas, and Prêtre, Phys. Lett. A, 180, 364 (1993).

[15] M. Büttiker, J. Phys.: Condens. Matter, 5, 9361 (1993).

[16] M. Büttiker, I. L. Nuovo Chimento, Vol. 110B, No. 5-6, 509 (1995).

[17] M. Büttiker, In: Quantum Dynamics of Submicron Structures, edited by H. A. Cerdeira et al., p.p. 657-672 (1995, Kluwer Academic Publishers, Netherlands);

W. Chen, T. P. Smith III, and M. Büttiker, Phys. Rev. Lett., 73, 146 (1994).

[18] T. Christen, M. Büttiker, Phys. Rev. Lett., 77, 143 (1996).

[19] T. Christen, M. Büttiker, Phys. Rev. B, 53, 2064 (1996).

[20] T. Christen, M. Büttiker, Europhys. Lett., 35(7) 523, (1996).

[21] H. Thomas, Prêtre, H. Thomas, and M. Büttiker, Phys. Rev. B, 54, 8130 (1996).

[22] C.L. Fernando and W.R. Frensley, Phys. Rev. B 52, 5092 (1995).

[23] L.Y. Chen and C.S. Ting, Phys. Rev. Lett. 64, 3159 (1990).

[24] C. Jacoboni and P.Price, Solid State Commun. 75, 193 (1990).

[25] Y. Fu and S.C. Dudley, Phys. Rev. Lett. 70, 3159 (1990); C. Jacoboni and P.J. Price, Phys. Rev. Lett. 71, 464 (1993); Y. Fu and S.C. Dudley, Phys. Rev. Lett. 71, 466 (1993).

[26] E. Wigner, Phys. Rev., 40, 749 (1932).

[27] A. I. Akhiezer and S. V. Peletminskii, Methods of statistical physics, (Oxford, New York, Pergamon Press, 1981).

[28] D.K. Ferry and H.L. Grubin, Modelling of Quantum Transport in Semiconductor De- 
vices, In: Solid State Physics, 49, 283 (1995);

N.C. Kluksdahl, A.M. Kriman, D.K. Ferry, and C. Ringhofer, Phys. Rev. B, 39, 7720 (1989);

J.-R. Zhou, D.K. Ferry, IEEE Transaction on Electron Devices, bf 39, 473 (1992).

[29] W.R. Frensley, Phys. Rev. B, 36, 1570 (1987).

[30] R. Landauer, IBM J. Res. Der., 1, 233 (1957); Phil. Mag., 21, 863 (1970);

R. Landauer,Z. Phys. B, 68, 217 (1987);

R. Landauer and T. Martin,Rev. Mod. Phys., 66, 217 (1994). 


\section{FIGURE CAPTIONS}

Fig. 1: The geometry of the quantum point contact. The width is denoted by $2 d(x)$, the narrowest width is $2 d_{0}$, and the effective length is $2 \tilde{L}$.

Fig. 2: The plane of phase trajectories for one-dimensional motion determined by the conservation of the integrals of motion. The heavy lines are separatrices that separate the propagating modes (regions 1 and 2) and non-propagating (reflecting) modes (regions 3 and $4)$.

Fig. 3: Equivalent circuit of the QPC. 


$$
H H
$$



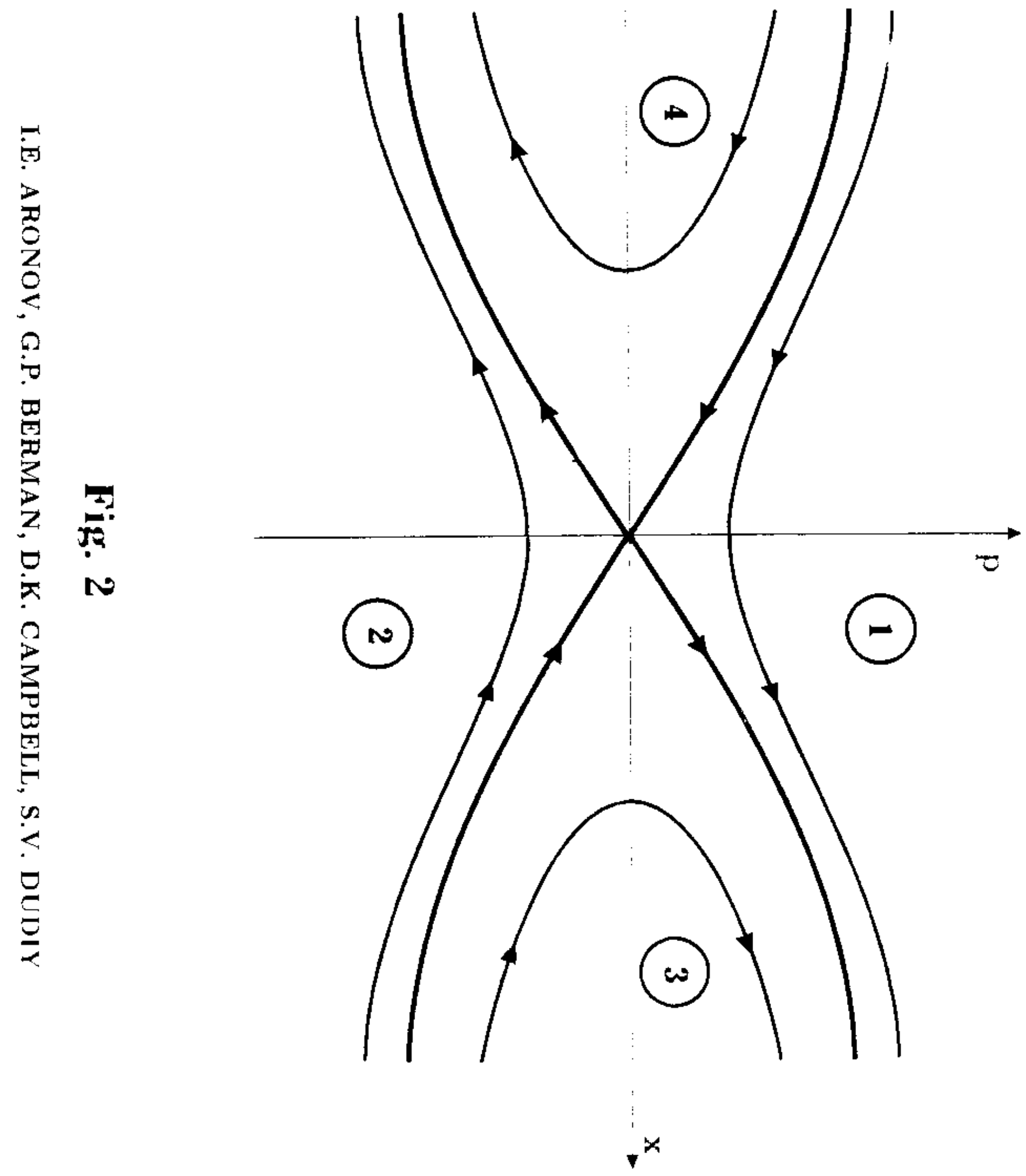

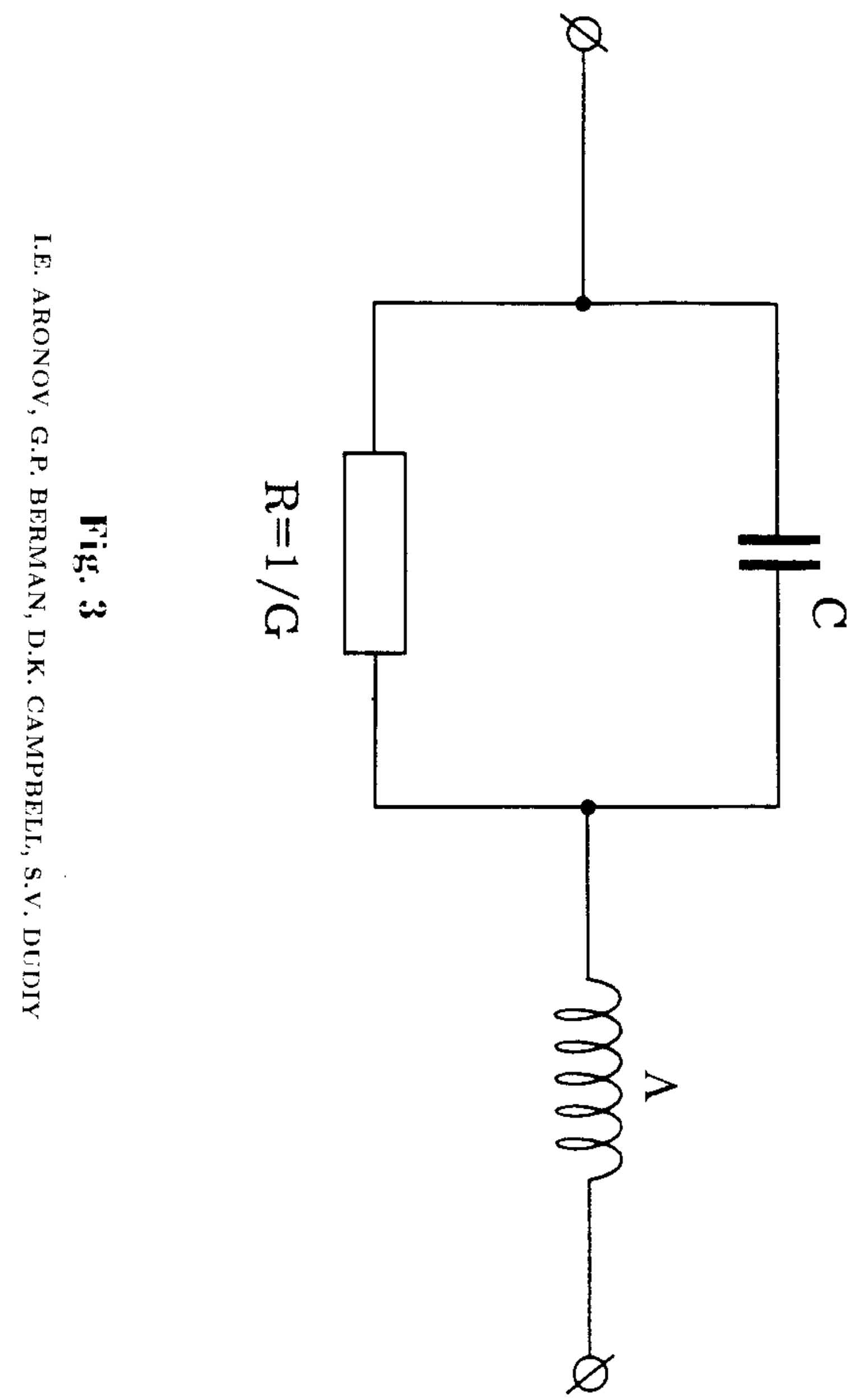